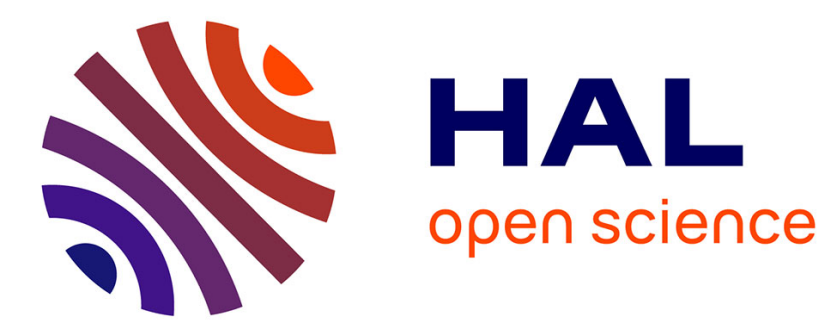

\title{
A comparison between Ambient Assisted Living Systems
}

\author{
Molham Darwish, Eric Senn, Christophe Lohr, Yvon Kermarrec
}

\section{To cite this version:}

Molham Darwish, Eric Senn, Christophe Lohr, Yvon Kermarrec. A comparison between Ambient Assisted Living Systems. ICOST 2014: 12th International Conference on Smart Homes and Health Telematics, Jun 2014, Denver, United States. pp.231 - 237, 10.1007/978-3-319-14424-5_26 . hal01186325

\section{HAL Id: hal-01186325 \\ https://hal.science/hal-01186325}

Submitted on 4 Sep 2018

HAL is a multi-disciplinary open access archive for the deposit and dissemination of scientific research documents, whether they are published or not. The documents may come from teaching and research institutions in France or abroad, or from public or private research centers.
L'archive ouverte pluridisciplinaire HAL, est destinée au dépôt et à la diffusion de documents scientifiques de niveau recherche, publiés ou non, émanant des établissements d'enseignement et de recherche français ou étrangers, des laboratoires publics ou privés. 


\title{
A comparison between Ambient Assisted Living Systems
}

\author{
Molham Darwish $^{1}$, Eric Senn ${ }^{2}$, Christophe Lohr ${ }^{1}$, Yvon Kermarrec ${ }^{1}$, \\ ${ }^{1}$ Ecole Nationale Supérieure des Télécommunications de Bretagne \\ Technopôle Brest-Iroise - CS 83818 - 29238 Brest Cedex 3 - France \\ \{molham.darwish, christophe.lohr, yvon.kermarrec\}@ telecom-bretagne.eu \\ ${ }^{2}$ Lab-STICC (CNRS), European University of Brittany, Université de Bretagne-Sud, \\ Centre de Recherche, BP 92116, F-56231 Lorient Cedex, France \\ eric.senn@univ-ubs.fr
}

\begin{abstract}
The growing number of older people emerges the need to consider autonomy concerns for those people and their need for intensive care. Plenty of researches, such as those in the domain of Ambient Assisted Living Systems, have been carried out to create enhanced conditions for older and people with disabilities, based on providing ICT solutions that enhance the well-being of elderly people and provide them with well independent daily living. In this paper, for better efficiency for such solutions, different essential requirements should be realized to meet those system objectives towards various users. These requirements are identified and introduced. Set of systems has been introduced with their solutions and provided services architecture, to reach the main goal of this work, by evaluating these systems from identified requirements perspective, by studying the functionalities of each system, if they meet the proposed requirements.
\end{abstract}

Keywords: Ambient Assisted Living, Interoperability, adaptability, reconfiguration, distributed access points.

\section{Introduction}

The number of elderly people will rapidly grow according to the new European population projections for 2008-2060 [1]. This growing number of older people will cause considerable issues for most European countries, since these aging people are living alone and need intensive care. Various researches have been carried out to provide ICT based solutions that enhance the well-being of elderly people and provide them with a well margin of independency in their daily life. Ambient Assisted living (AAL) systems aims to create improved conditions for older people as well as people with disabilities and reinforce the well-being of tem through the use of information and communication technology. These objectives are reached by 
providing different types of solutions for various users, which aim to enhance the quality of life for aging groups and take care of their health conditions. Therefore, in order to accomplish their objectives, the provided solutions should have specific features that increase systems efficiency towards user expectations. These features could, for instance, provide cost effective solutions with the consideration of user needs involvement, and privacy, as well as considering the participating of all related users, such as caregivers, family members and medical staffs, in the utilization of the system.

The contribution in this work is to identify a set of crucial features for Ambient Assisted Living systems to increase the idealism of these systems towards user needs and expectations, and surveying various projects to investigate what features they maintain in their functionalities, by studying systems architecture, included technologies and provided services.

This paper is organized as follows. Section (2) analyzes and defines the specific features of AAL systems and introduces the main requirements of an AAL system. Section (3) presents number of projects and systems that are developed under various programs and approaches, to provide ambient assisted living services for elderly people and people at home. Section (4) makes evaluation of the proposed systems in section 4 , according to the stated requirements defined in section 3 . The paper make conclusion in section (5).

\section{Ambient Assisted Living Systems}

Recent years, we are seeing the raise of techniques and technologies dedicated to make houses "intelligent", by providing different kinds of devices and services to people with disabilities (due to age or disease), which allow a more comfortable life and better safety [2].

Diverse researches have been conducted to use the latest technologies and facilities to enhance the well-being and support the independent living of older people at home, based on the notion of "ambient assisted living" (AAL), by the use of pervasive ICTs to enable the older persons to live autonomously in their own homes [3].

The AAL is an approach that aims to create better life conditions for older people and to reinforce the industrial opportunities in Europe through the use of information and communication technology (ICT), by providing more efficient and more productive solutions. According to [4], services provided by AAL systems can be categorized into emergency treatment services, autonomy enhancement services as well as comfort services which significantly improve the quality of life. To develop such a system, users and deployment environments have number of specificities that should be considered when an AAL system is developed, to fulfill the user requirements [5], as the capability of users, their acceptability, as well as target platforms capabilities of deployment environment and used devices configuration.

As the AAL systems considered as pervasive software systems [3], a number of non-functional requirements need to be considered in any system. Such nonfunctional requirements [6] are adaptability, interoperability, availability, distributed access points, maintainability, price, acceptability, etc... Some of these requirements are specific to home automation support systems [7], which can summarized by the following: 
- Adaptability: In definition, the ease with which a system or component can be modified for use in applications or environments other than those for which it was specifically designed [8]. As a crucial requirement for AAL systems, home automation services must address the specific needs of users, closely related to their disability, but also the configuration of their living space. In [9], different requirements of the various users, according to their capabilities and disabilities, in addition to the evolution of these requirements must be considered, as well as the evolution of the living space. This includes the ability of adapting the services dynamically without user intervention.

- Interoperability: According to [8], interoperability is the ability of two or more systems or components to exchange information and to use the information that has been exchanged. We must address the heterogeneity of technologies and communication protocols found in a home, as well as those who are to be integrated. Smart home environment includes various types of heterogeneous systems and services [10]. These heterogeneous systems are belonging to different middlewares [11], totally isolated from each other, and running with different technologies. The heterogeneity of systems considered as an essential challenge for the integration of all devices according to a standard mechanism that makes the heterogeneous systems interoperable and "speak the same language" apart from the used technology [11].

- Reconfiguration: Reconfiguration is defined in [12] as "reconfiguration is a process to perform changes to the system to ensure correct suitability to the current context. Changes can be made to the software, the hardware, or to both, and are triggered due to either objective changes (adaptively), or to service failures (faulttolerance)". The availability in case of failure is an essential feature that must be taken into account to ensure the delivery of a service [13]. In other words, it is the ability to overcome the failure of service implementation through the definition of different scenarios during the design time. Reconfiguration is performed using statically defined reconfiguration rules in resource descriptions [12], [13], or in a dynamic manner based on reconfiguration rules generated in execution time.

- Distributed access points: In [16], the degree to which a component, including devices, systems, resources or services, can be used by as many people as possible. The services provided by the AAL system need to be accessible from different access points, in order to make all users (medical staff, the elderly, the relatives) participate in using the services according to their roles [4].

What have been introduced in this section are some issues that are specific to the home automation assistance systems or AAL solutions, when other requirements are involved in the general acceptance of a home automation solution and related to the common approval of final users, which is efficient and effective towards elderly people needs, based on adding non-functional attributes to the ICT solution [17].

\section{Overview Of AAL Systems}

In recent years, the EU has funded different projects promoting independent living for elderly. As examples of these projects, the project funded by ICT Policy Support Programme under the Competitiveness and Innovation framework Programme (CIP) 
[18] and the Seventh (FP7 -2007-2013) Framework Programmes for Research and Technological Development [19].

The Ambient Assisted Living Joint Program (AALJP) [20] is focused exactly on this topic. The solutions funded by this program are categorized, according to the afforded solutions, into six key groups as stated in [20].

In this paper, four systems have been chosen to be introduced and to be evaluated according to specified requirements. Choosing of those systems are based on the well documentation provided with each of them, in which the functionalities and the architecture of each system are described, in addition to the scientific papers and publications related to each system, in which the objective and the services they provide are illustrated in details. The four projects are briefly described in the following sub sections.

\subsection{GiraffPlus}

GiraffPlus (January 2012- December 2014) is one of many running projects that are financed by the CIP and the FP7 [21]. This project addresses number of issues related to enhance the well-being of elderly people and extend their independency of living, by the early detection of possible health problems to decline them and providing services to assist those have deficiencies [22]. The main components of GiraffPlus system consists of a network of sensors that ubiquitously integrated in the home and categorized into physiological and environmental sensing devices. The collected data from these sensors are interpreted by an intelligent system which in turn prompts alarms or reminders for two kinds of users, the elderly users at home (primary users) and related caregivers and health professional (secondary users). The system consists also of telepresence robot, the Giraff robot, which is controlled over the internet by a remote user to move inside the home, in order to assist the elderly users to sustain their social contacts with other users. Giraff robot has an interface (similar to Skype) and equipped with communication facilities [22]. Further information about GiraffPlus can be obtained from [23].

\subsection{InCASA}

"Integrated Network for Completely Assisted Senior citizen's Autonomy (InCASA)" (April 2010 - September 2012) deals with citizen- centric technologies and public/ private services network, to help and protect independent elderly people, prolonging the time they can live well in their own home by increasing their autonomy and self-confidence [9], [24]. This project is also one of the projects that have funded by the CIP and the FP7.

The main objectives of InCASA project are to provide elderly people with solutions to monitor their health conditions when they are at their homes, to provide General Practitioners (GPs) and health professionals with technological facilities to remotely analyze and control elderlies' health conditions, and to ensure care continuity through the interaction between the elderly at home and his/her health professionals [9], [25], [26]. In order to meet its objectives, InCASA architecture is organized into three main tiers which purpose at providing integrated solutions and 
services to control the environment and health conditions for the elderlies and give feedback and alerts to the users. The three tiers are illustrated in [25].

\subsection{ECAALYX}

This project is one of many projects that has been developed and currently developed under the AALJP Programme. The AAL JP is "a funding activity that aims to create better condition of life for the older adults and to strengthen the industrial opportunities in Europe through the use of information and communication technology (ICT). It carries out its mandate through the funding of across-national projects (at least three countries involved) that involves small and medium enterprises (SME), research bodies and user's organizations (representing the older adults)" [27].

The project eCAALYX (Enhanced Complete Assisted Living Experiment) is a three-year project between 2009 and 2012. ECAALYX continued the advantages of the architecture and functionality that are developed by the project called CAALYX [27], [28], and aims to provide a solution in order to improve the well-being of elderly people at home and extend their independency for longer periods, by developing home and outdoors $24 / 7$ health telemonitoring / telehealthcare services for older people, including those with multiple chronic conditions. The key objective of eCAALYX project is [27], [28] to monitor the health of elderly people and people with chronic conditions, in order to improve their quality of life by strength their autonomy and safety, by providing continuous health support for elderly persons that assists in prolonging the need for intervention from health professionals and avoiding the descent of health circumstance. The project objectives could be accomplished by providing an ICT solution that meets all users' expectations.

The proposed ICT solution is based on an architecture, which consists of three main interconnected subsystems which are Home Subsystem, Mobile Subsystem and Caretaker Subsystem. This architecture is figured out with more details in [28].

\subsection{Danah}

This project is one of seven projects that are emerged by the team MOCS (Methods, Tools for Circuits and Systems) in the Lab-STICC laboratory in France. This team participated for many years in the development of home automation projects aiming to assist dependent people. The researches area of these projects is particularly "pervasive systems to help the handicaps" [7]. These projects are funded by the Bretagne region and the General Council of Morbihan.

The main objective of Danah project (2006) is to set out the different scientific issues from previous projects to improve the propositions that have been made [14]. The project proposed a middleware that integrates a process of multilevel reconfiguration, static and dynamic reconfiguration of home automation services, in addition to the topological reconfiguration of paths for the movement of an intelligent wheelchair. Danah middleware is based on client-server architecture (distributed system). Home automation terminals (Danah servers), are distributed in the environment [7], [12]. A terminal can thus be connected to a home automation bus KNX-EIB [15] to control equipments connected to it. Other terminals are responsible for monitoring equipment to local activation via infrared or Bluetooth such as 
multimedia equipment. The user, equipped with a PDA (Personal Digital Assistant) embedded in his wheelchair, has the opportunity to reach the services and methods published by these terminals.

\section{Evaluation Of The Systems}

Following the identification of AAL requirements in addition to the introduction of different AAL projects and knowing their architectures, this section, evaluate the previous projects from system requirements perspective; the four AAL solutions should be investigated to emphasize if they meet the general AAL requirements which have been mentioned in section (2). Those requirements again are: adaptability of services, interoperability of services, reconfiguration, and distributed access points.

- GiraffPlus is configured to have an important feature that makes the system adaptive in a way allowing for adding and removing services easily and related sensors that provide these services. In addition, the system is configured to accept the adaptation [22]. The system architecture consists of number of integrated functionalities; one essential part of system architecture is "Data Visualization, Personalization and Interaction Service" which is responsible for creating useroriented service. In addition, the system meets the requirement of distributed access points, since it includes not only the users at home, but also their family members and caregivers who are able to connect and control Giraff robot, as well as have access through PCs to the information and activities of the elderly people. This also applied for the medical stuff that are also can access the system to obtain health measurements [21]. GiraffPlus system architecture is designed by integrating a middleware infrastructure that hiding the heterogeneity of the computational resources used in the living space, since that the services provided by the system components are developed using different technologies and connecting with each other using different protocols.

- InCASA system is developed using an architecture that allows for the main blocks of the system to communicate regardless the used protocols or the coding modules [24], which in turns undertake the heterogeneity of protocols, which are used in the Body Sensor Network and in the Home Sensor Network [9]. The functional architecture of InCASA are realized using four levels of communication at home, with service center and with external users such as relatives, caregivers and general practitioners, which give different users of the system the ability to use the system and interact with it from different locations.

- eCAALYX home healthcare monitoring approach is performing the provided services depending on a system architecture that permits customization of the services, which makes these services adaptive to each user needs including the changing condition of the users at home [28], by reusing the Telecom remote management infrastructure as a unifying layer between different low-level sensor communication protocols and health communication standards. The interoperability between different system components is also underlined, since eCAALYX eHealth service interoperability approach "builds on Continua Version One standard and the Broadband Forum TR-069 CWMP specification" [28]. "Continua" is a defined standard that aim to develop ICT based healthcare systems following interoperability guidelines [29]. 
- Danah project is considered the reconfiguration of services in case of failure by defining a set of reconfiguration scenarios in the resource description. The developed middleware in the system, allows to deploy Danah in heterogeneous living space, regardless of the communication technology and used technologies, since the system uses different communication protocols to communicate clients with servers, such as KNX/EIB, X10, infrared, Bluetooth, and WiFi [15].

Table [1] summarizes the general AAL systems' requirements and how they are met by the four systems.

Table 1. Evaluation of AAL systems

\begin{tabular}{|c|c|c|c|c|}
\hline & Adaptability & Interoperability & Reconfiguration & Distributed Access Points \\
\hline GiraffiPlus & $\mathrm{X}$ & $\mathrm{X}$ & - & $\mathrm{X}$ \\
\hline InCASA & - & $\mathrm{X}$ & - & $\mathrm{X}$ \\
\hline eCAALXY & $\mathrm{X}$ & $\mathrm{X}$ & - & - \\
\hline Danah & - & $\mathrm{X}$ & $\mathrm{X}$ & - \\
\hline
\end{tabular}

\section{Conclusion}

An Ambient Assisted Living system is characterized by its functional and nonfunctional properties that it contains to meet its users expectations and needs. Increasing the efficiency and effectiveness of a system is achieved when it has as features as possible to reach it objectives. The main contribution of this paper is to evaluate number of Ambient Assisted Living Systems in order to characterize the main features that these systems have included. This evaluation based on identified requirements. By studying the different AAL systems according to the stated requirements, it is outlined that these systems meet the requirements partially. Thus, the attempt is to propose a system that meets all requirements so that it can be govern that the system meets all users' expectations. From this study, it is also stated that reconfiguration as an essential requirements is not considered systemically in most AAL systems, even though reconfiguration and failure tolerance in case of error occurrence, is considered a crucial requirement for distributes systems, especially those systems that deals with health conditions and assistance such as AAL systems.

\section{References}

1. http://epp.eurostat.ec.europa.eu/portal/page/portal/eurostat/home/

2. A. Helal, M. Mokhtari, B. Abdulrazak. The Engineering Handbook of Smart Technology for Aging, Disability and Independence. 2008. ISBN: 978-0-471-71155-1.

3. G.Virone, A. Sixsmith. Toward Information Systems for Ambient Assisted Living. In Proceedings of the 6th Int. Conference of the International Society for Gerontechnology, Pisa, Tuscany, Italy, June 4-7, 2008 
4. L. Jean-Baptiste et al. A design process enabling adaptation and customization of services for the elderly. Int. Workshop on Ambient Assisted Living, Valancia, Spain, 2010

5. L. Jean-Baptiste et al. Design process enabling adaptation in pervasive heterogeneous contexts. Personal and ubiquitous computing, pp 353-363, January 2011.

6. D. Schneider, M. Becker, Runtime Models for Self-Adaptation in the Ambient Assisted Living Domain. 3rd Workshop on Models@run.time at MODELS 2008. Proceedings: 30 September 2008, Toulouse, France.

7. W. Allegre. Flot de conception dirigé par les modèles pour la commande et la supervision de systèmes domotiques d'assistance. PhD thesis 2012, UNIVERSITE BRETAGNE SUD, Lorient.

8. 610.12-1990 - IEEE Standard Glossary of Software Engineering Terminology. Dec. 311990

9. G. Lamprinakos. An Integrated Architecture for Remote Healthcare Monitoring. 14th Panhellenic Conference on Informatics 2010.

10.[18] T. Perumal, A. R. Ramli, C. Y. Leong, S. Mansor, K. Samsudin. Interoperability among Heterogeneous Systems in Smart Home Environment. 2008 IEEE International Conference on Signal Image Technology and Internet Based Systems

11.V. Miori, L. Tarrini, M. Manca. An Open Standard Solution for Domotic Interoperability. Consumer Electronics, IEEE Transactions on (Volume: 52, Issue: 1). Feb2006.

12. S. Lankari, Jean-Luc PHILIPPE. Multi-Level Reconfiguration in the DANAH Assistive System. In Proceedings of the IEEE International conference on Systems, Man and Cybernetics. San Antonio, USA. 2009.

13.S. Lankari, et al. Service Reconfiguration in the DANAH Assistive System. In Proceedings of the 7th International Conference on Smart Homes and Health Telematics. France 2009.

14. S. Lankri, P. Berruet, A. Rossi Jean-Luc Philippe. Architecture and Models of the DANAH Assistive System. SIPE '08 Proceedings of the 3rd international workshop on Services integration in pervasive environments.

15.S. Lankri. Services et Navigation pour Personnes Dépendantes en Environnements Domotiques. PhD thesis 2009, UNIVERSITE DE BRETAGNE SUD, Lorient.

16.A. Queirós et al. Usability, accessibility and ambient-assisted living: a systematic literature review. Universal Access in the Information Society. October 2013.

17.H. Sun, V. D. Florio, N. Gui, C. Blondia. The Missing Ones: Key Ingredients towards Effective Ambient Assisted Living Systems. Journal of Ambient Intelligence and Smart Environments, Volume 2, number 2, 2010. P.109-120.

18.http://ec.europa.eu/information_society/activities/ict_psp/index_en.htm

19.http://cordis.europa.eu/fp7/ict/

20.http://www.aal-europe.eu/

21.S. Coradeschi et al. GiraffPlus: Combining social interaction and long term monitoring for promoting independent living. HSI 2013.

22. A. Cesta et al. Steps Toward End-to-End Personalized AAL Services. Workshop Proceedings of the 9th International Conference on Intelligent Environments 2013.

23.http://www.giraffplus.eu/

24.A. Prestileo, R. di Fiore. inCASA project-Smart telemonitoring. TeleMediCare 2012.

25.G. V. Lioudakis. Introducing Privacy-Awareness in Remote Healthcare Monitoring. Applied Sciences in Biomedical and Communication Technologies (ISABEL), 2010.

26.M2M Journal, ISSN 1868 - 9558, March 2013. Page 11.

27.M.N.K Boulos et al. Connectivity for Healthcare and Well-Being Management: Examples from Six European Projects. Int. J. of Environmental Research and Public Health 2009.

28.M.N.K Boulos. ECAALYX: Towards a Real-world Ambient Assisted Living Solution that Delivers in Non-technical Environments and Is Sustainable. 2009

29.R. Carroll, et al. Continua: An Interoperable Personal Healthcare Ecosystem. IEEE_Pervasive_Computing. Vol. 6, No. 4 October-December 2007. 